\title{
The epithelial flap for photorefractive keratectomy
}

\author{
S Shah, A R Sebai Sarhan, S J Doyle, C T Pillai, H S Dua
}

\begin{abstract}
Backgroundlaims-Epithelial debridement for photorefractive keratectomy (PRK) is associated with pain, slower visual recovery, and may be aetiological in haze production. The aim of this study was to assess the clinical results of a new technique involving raising and replacing of an epithelial flap in photorefractive keratectomy.
\end{abstract}

Methods-A prospective, non-randomised, comparative, paired eye trial was performed in 72 eyes of 36 patients who underwent PRK with a Nidek EC-5000 excimer laser. For epithelial debridement before PRK, the eyes were divided into two groups. The first eye of each patient was treated with $20 \%$ ethanol debridement and the second eye with an epithelial flap which was replaced after treatment. PRK was carried out with the same laser and nomogram in both groups by the same surgeon. Visual and refractive outcome of PRK treatment was compared in both groups.

Results-The mean (SD) preoperative mean spherical equivalent (MSE) was -3.61 (1.38) dioptres (D) (range $-1.00 \mathrm{D}$ to $-7.88 \mathrm{D}$ ) with no significant difference between the two groups. After a mean follow up period of 62.6 weeks (range 52-70) the final MSE was $+0.07(0.61) \mathrm{D}$ (range $-5.50 \mathrm{D}$ to $+4.50 \mathrm{D})$ in the debridement group and $-0.24(0.43) \mathrm{D}$ in the epithelial flap group. There was no statistically significant difference between the two groups in postoperative MSE. The best corrected visual acuity was better in the
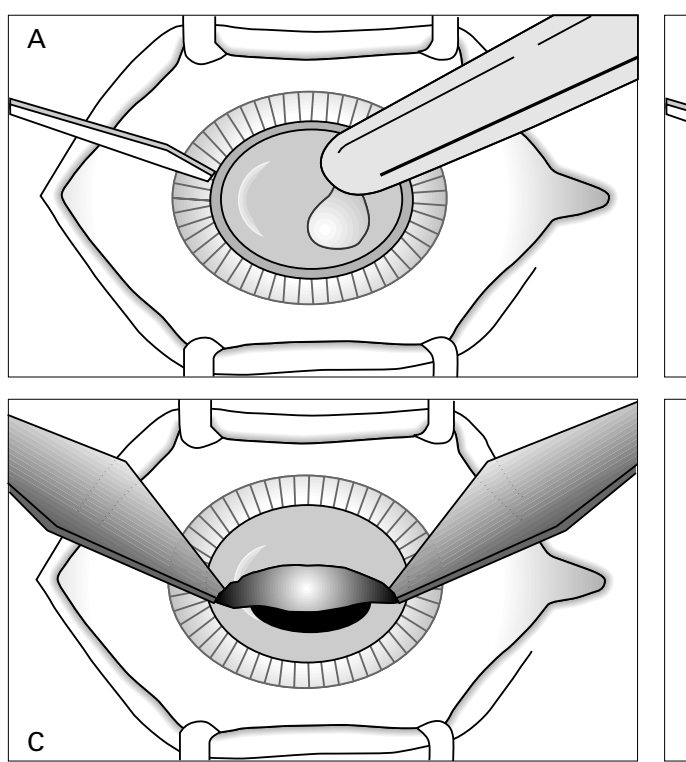

epithelial flap group at all visits; this difference was statistically significant $(p<0.05)$. The corneal haze was less in the epithelial flap group and this difference was also statistically significant $(p<0.05)$. Conclusions-Managing the corneal epithelium as a hinged flap with $20 \%$ ethanol is a safe technique with faster visual rehabilitation and reduced haze compared with debridement of the epithelium with alcohol. Further studies need to be performed to compare pain levels postoperatively with the epithelial flap and epithelial debridement.

(Br F Ophthalmol 2001;85:393-396)

Excimer laser photorefractive keratectomy (PRK) has become a popular surgical option for the treatment of myopia. Hundreds of thousands of eyes have been treated worldwide. Several techniques of epithelial debridement have been tried including mechanical debridement, ${ }^{1}$ laser transepithelial ablation, ${ }^{23}$ a rotating brush, ${ }^{4}$ and alcohol debridement. ${ }^{5}$ All of these techniques result in a bare corneal surface which takes 2-3 days to re-epithelialise and another 2 days for the epithelium to settle. Epithelial debridement is not without problems. It is associated with pain which is maximal in the first $24-48$ hours and continues until the epithelium heals completely. Visual recovery is delayed until epithelial healing is complete, and the healing process is likely to play a significant role in the production of haze. ${ }^{6}$ We hypothesised that a corneal epithelial flap, lifted before laser application,

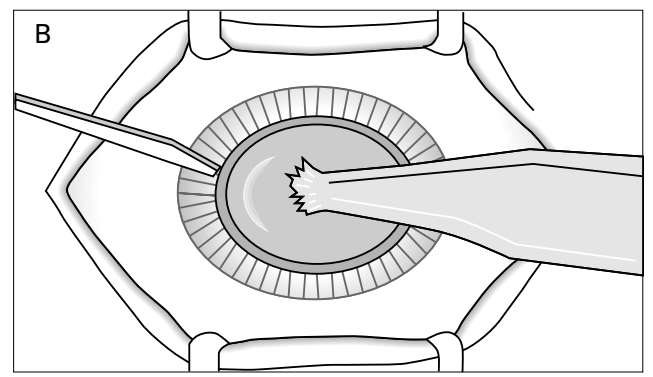

C T Pillai

H S Dua

Optimax Laser Eye Clinics, Manchester, UK

S Shah

S J Doyle

Correspondence to: Dr S Shah, Birmingham Heartlands Hospital, Bordesley Green East, Birmingham B9 5SS, UK sunilshah@doctors.net.uk

Accepted 27 November 2000

Figure 1 Technique for raising an epithelial flap. (A) Application of 20\% ethanol in a $9 \mathrm{~mm}$ ring marker. (B) Absorbing ethanol with mercel sponge. (C) Lifting epithelial flap with two mercel sponges. (D) Superior hinged flap prior to PRK. 


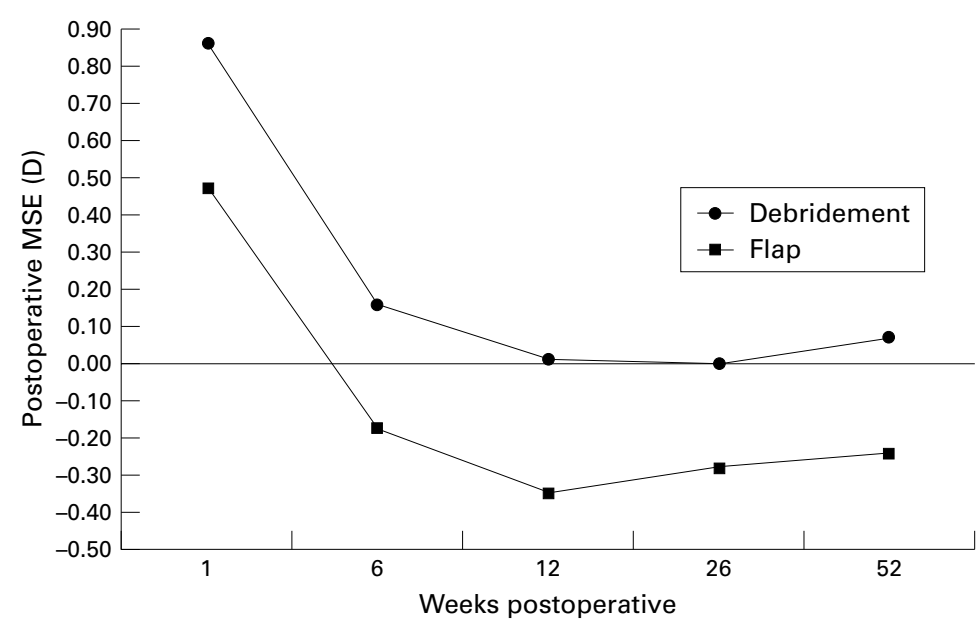

Figure 2 Mean spherical equivalent (MSE) in dioptres (D) at different postoperative periods in the ethanol debridement group and the epithelial flap group.

would enhance visual recovery and reduce haze production.

\section{Materials and methods}

This was a consecutive case study involving 72 eyes of 36 patients treated with PRK for correction of myopia. For each eye the mean spherical equivalent (MSE), the unaided visual acuity (UCVA), and the best corrected visual acuity (BCVA) (Snellen fractions) before PRK and at the most recent follow up visit were assessed. The presence of corneal haze was recorded at the latest follow up. Corneal haze was assessed by slit lamp examination and recorded on a qualitative scale of $0-3(0=$ clear cornea; $1=$ mild haze not interfering with refraction, $2=$ moderate haze, $3=$ marked haze obscuring iris detail).

The first eye underwent epithelial debridement with $20 \%$ ethanol and the epithelium was discarded. In the second eye the epithelium was raised as a flap using the new technique and, following PRK, the epithelium was replaced as described below. The procedure was performed by the same surgeon using the same nomogram with the Nidek EC-5000 excimer laser (Nidek Co Ltd, Aichi, Japan). Informed consent was obtained. PRK was performed with a $6.5 \mathrm{~mm}$ optical zone and an

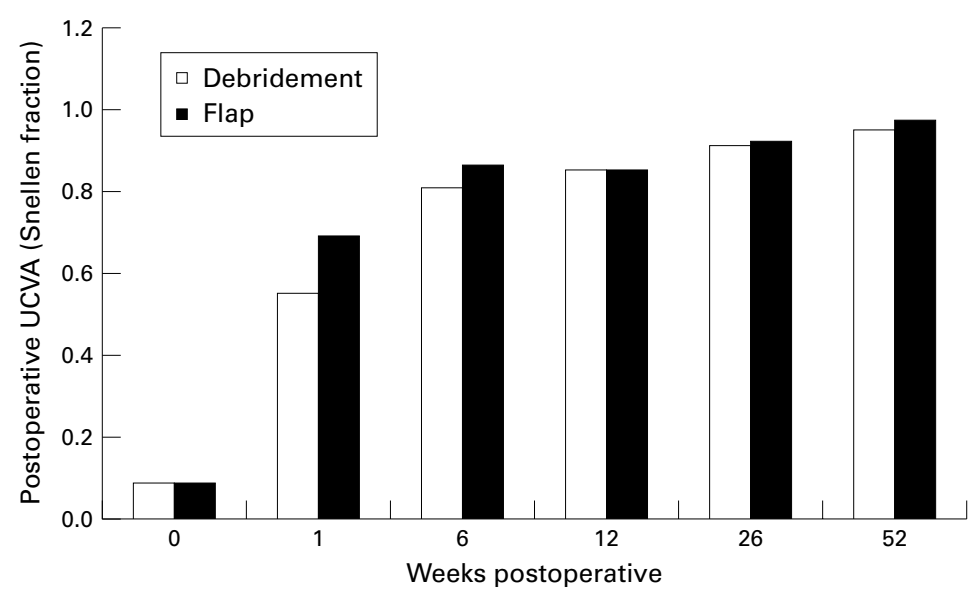

Figure 3 Mean uncorrected visual acuity (UCVA) at different postoperative periods in the ethanol debridement group and the epithelial flap group. additional $1.0 \mathrm{~mm}$ transition zone. Postoperatively, topical chloramphenicol drops (5 days) and topical non-steroidal anti-inflammatory agents (NSAID) (diclofenac sodium $0.1 \%$, Ciba Vision, Southampton, UK) for 2 days were used routinely. No topical steroids were used.

\section{TECHNIQUE}

Group 1

Epithelial debridement was carried out using a $9 \mathrm{~mm}$ corneal ring marker. $20 \%$ alcohol was placed inside the marker for 30 seconds (being careful to avoid spillage on the areas not being treated) and the ethanol was then absorbed by a mercel sponge, washed with topical hypromellose drops, and an NSAID was applied. The surface was dried and the loose epithelium was peeled off the surface (by epitheliorhexis) using a dry mercel sponge. The surface was not dried any further. PRK was performed, antibiotics and NSAIDs were applied topically, and a double pad was placed on the eye for 6 hours.

\section{Group 2}

The epithelial flap involved the application of a $9 \mathrm{~mm}$ ring marker filled with $20 \%$ ethanol and left for 45 seconds. The ethanol was absorbed with a mercel sponge (being careful to avoid spillage on the areas not being treated), the cornea was washed with topical hypromellose, and an NSAID agent applied as above. A superiorly hinged epithelial flap was fashioned by lifting the edge of the loosened epithelium with a sharp beaver blade from 3 to 9 o'clock inferiorly. The epithelium was then rolled as a flap to the 12 o'clock position with a dry mercel sponge. The laser was applied on the bed created. The ablated bed was then dried and the epithelium rolled down from the 12 o'clock position with a mercel sponge. The epithelium was left to dry and to adhere to the ablated bed for 1 minute and a topical antibiotic and an NSAID were applied. The eye was not patched. This technique is shown in Figure 1.

\section{DATA ANALYSIS}

Data analysis was performed using spss Version 8.0 for Windows and statistical significance was tested using repeated measures analysis of variance.

\section{Results}

All patients had a minimum follow up of 52 weeks $(n=36)$ and there was $100 \%$ follow up for the duration of this study. Mean follow up was 62.6 weeks (range 52-70). The refractive and visual results of the PRK treatment are presented in Figures 2-5. The mean (SD) preoperative mean spherical equivalent (MSE) was $-3.73(1.42) \mathrm{D}$ (range $-1.38 \mathrm{D}$ to $-6.88 \mathrm{D}$ ) for group 1 and $3.49(1.36) \mathrm{D}$ (range $-1.00 \mathrm{D}$ to $-6.10 \mathrm{D}$ ) for group 2 . There was no significant difference between the mean preoperative MSE, sphere or cylinders of the two eyes of each patient. The mean (SD) age of this group was 33.5 (6.5) years (range 21-66). There were 20 female patients and 16 male. 


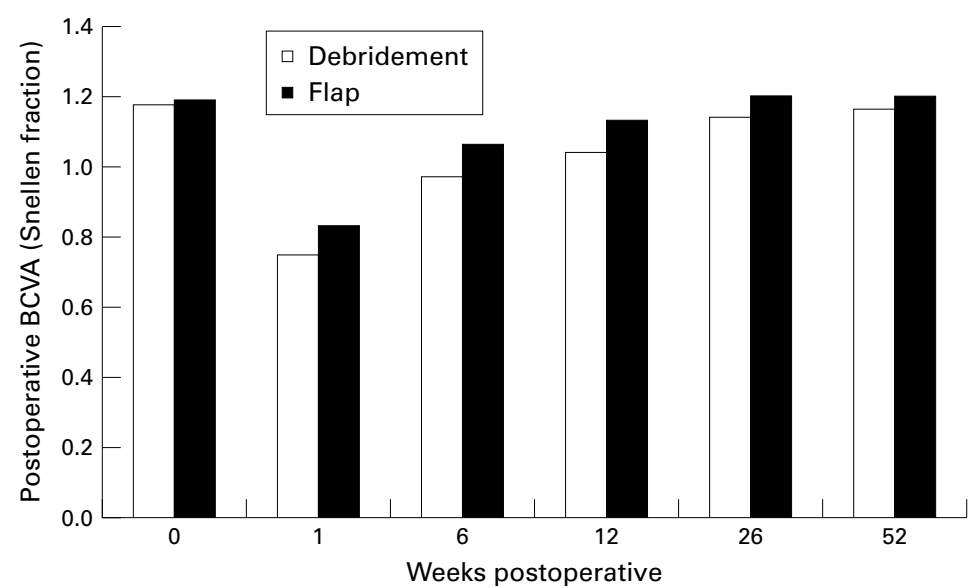

Figure 4 Mean best corrected visual acuity (BCVA) at different postoperative periods in the ethanol debridement group and the epithelial flap group.

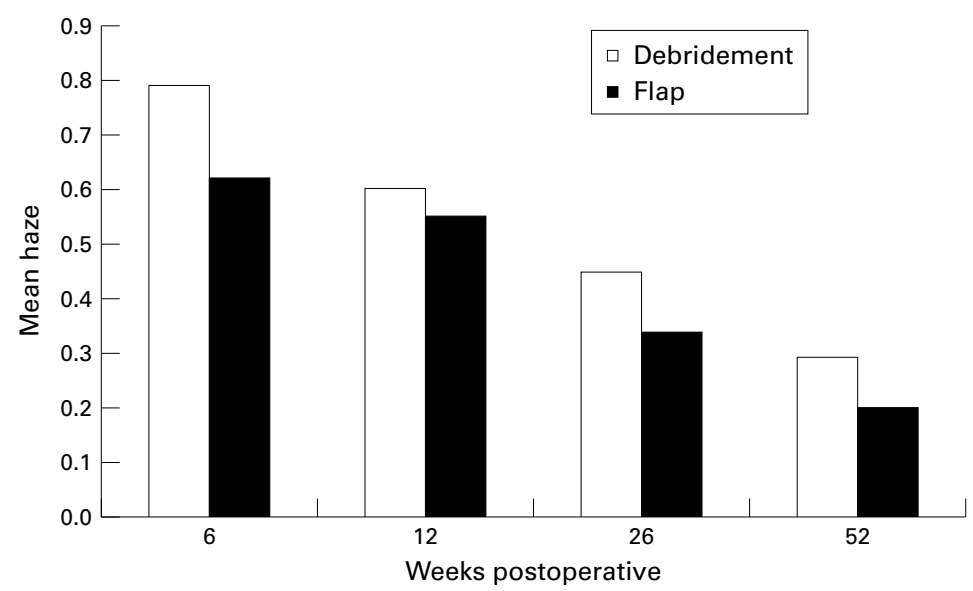

Figure 5 Mean haze (scale 0-3) at different postoperative periods in the ethanol debridement group and the epithelial flap group.

No eyes in this study suffered from flap related problems such as tearing or nonadherence.

REFRACTIVE DATA

The final MSE measured at 52 weeks was 0.07 (0.30) D for group 1 and $-0.24(0.43) \mathrm{D}$ for group 2. The postoperative refractive results are shown in Figure 2. There was no statistically significant difference between the two groups in the final MSE (repeated measures ANOVA).

\section{VISUAL ACUITY}

The final BCVA was 1.16 (0.13) (Snellen fraction) for group 1 and 1.20 (0.14) for group 2. The final UCVA was $0.95(0.22)$ for group 1 and $0.97(0.30)$ for group 2 . The postoperative visual results are presented in Figures 3 and 4. No statistically significant difference was found for UCVA, but BCVA was significantly better in the epithelial flap group $(\mathrm{p}<0.05$, repeated measures ANOVA). Nine eyes in the debridement group and five in the epithelial flap group lost one Snellen line of BCVA. No eyes in either group lost two or more lines of BCVA.

\section{CORNEAL CLARITY}

The mean (SD) haze at 52 weeks, recorded on a scale of $0-3$, was $0.29(0.32)$ for group 1 and $0.20(0.34)$ for group 2. Mean haze is shown in
Figure 5. Haze was significantly reduced in the epithelial flap group $(\mathrm{p}<0.05$, repeated measures ANOVA).

\section{Discussion}

The use of corneal epithelium as a flap to cover the ablated stromal bed after PRK resulted in significant improvement in the BCVA and also significantly reduced the corneal haze after the procedure in comparison with an alcohol epithelial debridement technique.

The common problems of epithelial debridement after PRK include moderate to severe pain, a relatively long period of visual recovery, and corneal haze. This study assessed the results of using an epithelial flap to reduce these problems and to maintain the safety of PRK in correction of low to moderate myopia. The described technique is relatively simple and produces highly predictable results. Using the corneal epithelium to cover stroma after laser application should theoretically reduce pain and allow for rapid epithelial healing as well, and may reduce corneal haze. The learning curve for the epithelial flap is steep. The main problems are reattachment of the flap if the surgeon has difficulty raising the flap, damage/tearing of the flap during manipulation, drying of the flap, and non-adherence of the flap. However, problems that can occur with the flap such as tearing or non-adherence leave an area which is effectively the same as if the epithelium had been debrided. All eyes in this study were operated on by a surgeon experienced in the epithelial flap technique and no intraoperative or postoperative flap complications such as dislocation, folds, central epithelial defects, or infiltrates occurred. The epithelium appeared to be viable postoperatively, covering the majority of the cornea and remaining in situ rather than sloughing off. For the purposes of this study, no contact lenses were used postoperatively. However, the authors have since begun to use soft contact lenses routinely for a period of 4 days as patients are more comfortable with this.

Several techniques of epithelial debridement have been tried including mechanical debridement, ${ }^{2}$ laser transepithelial ablation, ${ }^{3}{ }^{4}$ a rotating brush, ${ }^{5}$ and alcohol debridement. ${ }^{7}$ All of these techniques are reported to be effective in epithelial debridement. However, a fast and safe method of epithelial removal is essential. A smooth Bowman's membrane is believed to be important in obtaining a successful outcome from PRK. Manual epithelial debridement was found to produce scratches and nicking in the Bowman's membrane and to leave varying amounts of epithelium. ${ }^{89}$ Abad et $a l^{10}$ found that chemical de-epithelialisation with dilute ethanol $(18 \%)$ appears to be a safe and effective alternative to mechanical scraping before PRK and might promote faster rehabilitation. The same results were reported by Stein et $a l^{11}$ who used dilute alcohol (25\%) in 91 cases of PRK and found the technique to be effective, predictable, and safe with no significant loss of BCVA, no toxic effects, and no stromal dehydration. 
However, higher concentrations of ethanol such as $50 \%{ }^{12}$ and $100 \%{ }^{13}$ were found to cause inflammation and damage to the underlying stromal keratocytes. Griffith et $a l^{9}$ found that alcohol produced a smooth stromal surface but left some of the epithelial cells and needed wiping of the surface to remove all the remnant cells. The Amoils rotating brush has also been found to be a quick, effective and safe method to remove the corneal epithelium before PRK with minimal risk of damage to Bowman's layer. ${ }^{9}$

Removal of the corneal epithelium was found to cause damage of the stromal keratocytes $^{14}$ and these changes start within 15 minutes $^{15}$ to 30 minutes $^{14}$ of deepithelialisation in rabbit and monkey corneas. Other studies ${ }^{16}{ }^{17}$ have shown that an early decrease in the density of keratocytes is followed by an increased number of these cells in the underlying stroma and production of collagen and extracellular matrix. This regrowth is felt to be related to the subepithelial haze formation after PRK. There is evidence to suggest that keratocyte changes are influenced by the regenerating epithelium (cytokines). ${ }^{6}$ Covering of the denuded surface of the cornea directly after PRK with the corneal epithelial flap may decrease the changes in the stromal keratocytes and the production of extracellular matrix and collagen. This may explain the significantly reduced haze in the epithelial flap group in the present study.

As dilute alcohol was shown to be effective and safe for epithelial debridement, ${ }^{10}$ the present study compared two techniques using dilute ethanol to remove the corneal epithelium before PRK. The only difference between the two techniques was the re-use of the eye's own corneal epithelium as a flap to cover the stromal bed after PRK. There was a significantly better BCVA and significantly reduced haze in the epithelial flap group than in the ethanol debridement group.

The better UCVA in the epithelial flap group could be explained partly by the reduced haze in this group. The postoperative MSE was comparable in both groups although there was a small undercorrection in the epithelial flap group. If larger studies confirm this undercorrection, then the laser algorithm will have to be adjusted to allow for this.

In conclusion, use of corneal epithelium as a hinged flap to cover the denuded corneal surface after PRK was a safe and effective method of managing the corneal epithelium. There was a trend towards faster visual rehabilitation and reduced haze compared with debridement of the epithelium with alcohol (statistically significant for BCVA and haze). This technique may change the MSE threshold for treating eyes with LASIK rather than PRK. Further studies need to be performed to compare postoperative pain levels with the epithelial flap and epithelial debridement and to determine the extent of regression occurring over time. Presented in part at the British Society of Refractive Surgery
Meeting, Cambridge, UK in March 1999 and at the European Society of Cataract and Refractive Surgeons, Vienna, Austria in September 1999.

The authors acknowledge the doctors at Optimax Laser Eye Clinics in developing the epithelial flap and putting it into routine use, especially Drs S J Doyle, E F Hynes, A Nylander, and E R Dingley.

Dr Sunil Shah was the Vision Express Cornea and Contact Lens Fellow, Nottingham at the time of writing.

Lens Fellow, Nottingham at
Proprietary interests: none.

1 Gimbel HB, Levy SG. Indications, results and complications of LASIK. Curr Opin Ophthalmol 1998;9:3-8.

2 Sealer T, Wollensak J. Myopic photorefractive keratectomy with excimer laser: one-year follow-up. Ophthalmology 1991;98:1156-63.

3 Alio JL, Ismael MM, Artola A. Laser epithelial removal before photorefractive keratectomy (letter). Refract Corneal Surg 1993;9:395.

4 Gimbel HV, DeBroff BM, Beldavs RA, et al. Comparison of laser and manual removal of corneal epithelium for photorefractive keratecteomy. I Refract Surg 1995;11:3641

5 Palliarkis IG, Karoutis AD, Lydatki SE, et al. Rotating brush or fast removal of corneal epithelium. $\mathcal{F}$ Refract Corneal Surg 1994;10:439-42.

$6 \mathrm{Li} \mathrm{D}$, Tseng SCG. Three patterns of cytokine expression potentially involved in epithelial-fibroblast interactions of human ocular surface. f Cell Physiol 1995;163:61-79.

7 Aron-Rosa DS, Colin J, Aron B, et al. Clinical results of excimer laser photorefractive keratectomy: a multicenter

study of 265 eyes. F Cataract Refract Surg 1995;21:644-52. after deepithelialisation using a sharp and a dull instruafter deepithelialisation using a sharp and

9 Griffith M, Jackson B, Lafontaine MD, et al. Evaluation of current techniques of corneal epithelial removal in hyperopic photorefractive keratectomy. $\mathcal{F}$ Cataract Refract Surg 1998;24:1070-8.

10 Abad JC, Talamo JH, Vidaurri-Leal J, et al. Dilute ethanol versus mechanical debridement before photorefractive keratectomy. F Cataract Refract Surg 1996;22:1427-33.

11 Stein HA, Stein RM, Price C, et al. Alcohol removal of the epithelium for excimer laser ablation: outcomes analysis. $\mathcal{F}$ Cataract Refract Surg 1997;23:1160-3.

12 Campos M, Raman S, Lee M, et al. Keratocyte loss after different methods of de-epithelialisation. Ophthalmology 1994;101:890-4.

13 Helena MC, Filatov V, Johnston WT, et al. Effects of 50\% ethanol vs mechanical epithelial debridement on keratocyte loss and inflammatory response after excimer photorefractive keratectomy. Invest Ophthalmol Vis Sci 1995;36(suppl S24).

14 Campos M, Szerenyi K, Lee M, et al. Keratocyte loss after corneal de-epithelialisation in primates and rabbits. Arch Ophthalmol 1994;112:254-60.

15 Nakayasu K. Stromal changes following removal of the epithelium in rat cornea. $\mathcal{F p n} \mathcal{f}$ Ophthalmol 1988;32:113-25.

16 Hirst LW, Kenyon KR, Fogle JA, et al. Comparative studies of the corneal surface injury in the monkey and rabbit. Arch Ophthalmol 1981;99:1066-73.

17 Kenyon KR, Berman L, Rose J, et al. Prevention of stromal ulceration in the alkali-burned rabbit cornea by glued-on contact lenses: evidence for the role of polymorphnuclear leucocytes in collagen degradation. Invest Ophthalmol Vis Sci 1979;18:570-87. 